Check for updates

The BMJ

Cite this as: $B M / 2021 ; 372: n 186$ http://dx.doi.org/10.1136/bmj.n186 Published: 21 January 2021

\section{Covid-19: Arthritis drug trial for severe illness is stopped early after increase in deaths}

\author{
Elisabeth Mahase
}

A trial testing whether the arthritis drug tocilizumab plus standard care could improve outcomes for patients with severe or critical covid-19 has been stopped early after an increase in deaths. ${ }^{1}$

Just 129 patients (average age 57; 68\% male) had been enrolled in the trial when the data monitoring committee recommended that it should be stopped. This was due to 11 of 65 patients $(17 \%)$ in the tocilizumab group dying by day 15 , compared with two of $64(3 \%)$ in the standard care only group (odds ratio 6.42 (95\% confidence interval 1.59 to 43.2$)$ ).

The trial results, published in The $B M J,{ }^{1}$ seem to contradict earlier observational studies that reported a benefit of tocilizumab. Recent results from the REMAP-CAP trial showed that, when given within 24 hours of patients entering intensive care in addition to a corticosteroid, tocilizumab (given to 353 patients) reduced mortality when compared with standard care only (including corticosteroids). ${ }^{2}$

Tocilizumab blocks a specific part of the immune system (interleukin 6) that can go into overdrive in some patients with covid-19. Researchers hypothesised that this could help lessen the body's inflammatory response to the virus and avert some of the more dire consequences of the disease.

\section{Statistical significance}

To test this, researchers in Brazil conducted a randomised controlled trial from 8 May to 17 July 2020 in hospital inpatients who were receiving supplemental oxygen or mechanical ventilation and had abnormal levels of at least two chemicals linked to inflammation in their blood. Patients were randomly assigned to receive either tocilizumab plus standard care or standard care alone, and all patients were monitored for 15 days.

By day 15,18 patients ( $28 \%$ ) in the tocilizumab group and $13(20 \%)$ in the standard care group were receiving mechanical ventilation or had died (1.54 (o.66 to 3.66); $\mathrm{P}=0.32$ ). In both groups deaths were attributed to covid-19 related acute respiratory failure or multiple organ dysfunction. Adverse events were reported in 29 of 67 patients (43\%) who received tocilizumab and in 21 of 62 (34\%) who did not receive tocilizumab.

Martin Landray, professor of medicine and epidemiology at the University of Oxford and joint chief investigator for the RECOVERY trial, said that the study was small and that the difference in deaths was not statistically significant.

"It is important to consider the totality of the evidence: in total just over 2000 patients with covid-19 have been enrolled in published trials of tocilizumab, including REMAP-CAP,” he said. “Taken together, these trials suggest that there may be a modest reduction in the risk of death of about one sixth. But this is not statistically significant. It is also uncertain whether the presence or extent of any beneficial effect varies in different types of patient-for example, those requiring invasive mechanical ventilation versus those requiring oxygen alone.

“The RECOVERY trial has now randomised over 3500 patients with covid-19 tocilizumab versus usual care alone, including large numbers of patients requiring artificial ventilators. Recruitment is ongoing, and results are anticipated during the course of the next few weeks."

The paper authors noted a number of limitations to their study, including that the level of respiratory support-an important prognostic marker-was lower among patients assigned to tocilizumab. They added that the sample size was relatively small and so the 95\% confidence interval around the effect on the primary outcome was wide, “compatible with odds ratios between 0.66 (benefit of tocilizumab) and 3.66 (harm with tocilizumab)."

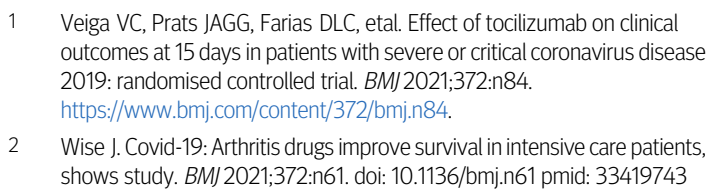

Veiga VC, Prats JAGG, Farias DLC, etal. Effect of tocilizumab on clinical outcomes at 15 days in patients with severe or critical coronavirus disease 2019: randomised controlled trial. BMJ2021;372:n84. https://www.bmi.com/content/372/bmi.n84.

2 Wise J. Covid-19: Arthritis drugs improve survival in intensive care patients, shows study. BM/2021;372:n61. doi: 10.1136/bmj.n61 pmid: 33419743

This article is made freely available for use in accordance with BMJ's website terms and conditions for the duration of the covid-19 pandemic or until otherwise determined by BMJ. You may use, download and print the article for any lawful, non-commercial purpose (including text and data mining) provided that all copyright notices and trade marks are retained. 